\title{
Hyperbaric oxygen therapy: a review of possible new era in dentistry
}

\begin{abstract}
Hyperbaric oxygen therapy is becoming widely used in various medical conditions. The list of its approved medical indications is getting longer by the Undersea and Hyperbaric Medical Society. On the other hand, the dentists predict it to become a new era that could help them for bone regeneration and to treat the challenging cases of wound healing and bacterial infections. This review article is shedding a light on how the HBO therapy developed, how it works physiologically and how it is used, its effectiveness in treating many conditions as adjunct or sole treatment, its possible applications in dentistry, and contraindications of HBO therapy, oxygen toxicity and ozone vs. HBO therapies.
\end{abstract}

Volume 9 Issue 2 - 2018

\section{Somaia Salah Meligy, Saaid Ayesh AI Shehadat, AR Samsudin}

College of Dental Medicine, University of Sharjah, UAE

Correspondence: Somaia Salah Meligy, College of Dental

Medicine, University of Sharjah, UAE,

Email somaia-meligy@hotmail.com

Received: March 09, 2018 | Published: April 12, 2018

\section{Introduction}

Hyperbaric oxygen (HBO) therapy has been defined by the Undersea and Hyperbaric Medical Society (UHMS), as an intermittent breath of $100 \%$ of oxygen, while the treatment chamber is pressurized to a pressure greater than the atmospheric pressure at sea level (more than 1 ATM). It is an approved medical application with widely increasing indications to treat various conditions including but not limited to, treatment of carbon monoxide poisoning, smoke inhalation, and enhancement of healing in selected problem wounds, necrotizing soft tissue infections and exceptional blood loss anemia. ${ }^{1}$ HBO therapy has significant physiological improvements for wound healing. Its effect was interpreted by amplifying oxygen gradients along the periphery of ischemic wounds, and promoting oxygendependent collagen matrix formation needed for angiogenesis., ${ }^{2,3}$ In addition, HBO has antibacterial effects by increasing generation of oxygen free radicals, which oxidize proteins and membrane lipids, damaging DNA of the bacteria and inhibiting the bacterial metabolic functions. It is effective particularly against anaerobes and facilitates the oxygen-dependent peroxidase system by which leukocytes kill bacteria. It also improves the oxygen-dependent transport of certain antibiotics across the bacterial cell walls. ${ }^{4,5}$ besides the medical applications, HBO has shown a good prognosis in various dental conditions. However, the application of $\mathrm{HBO}$ in dentistry requires more investigation. History of HBO therapyIt all started in 1662 before discovering the oxygen, when Dr. Henshaw, an English physician, built the first Hyperbaric Chamber by a sealed room called "domicilium". Since, the pressure was raised or lowered by a pair of organ bellows and series of valves. ${ }^{6}$ Ever since the 19th century and so on, the reported therapeutic effects of hyperbaric chamber were increasing and applied for treating various medical conditions. In the late 1880s, J. Leonard Corning, New York neurologist was the first to introduce "Compressed air paths" in the United States. The hyperbaric chamber was here operated with electricity powered air compressor. The resulted hyperbaric air was used as a facilitator in the treatment of nervous and mental conditions. After the First World War, using the hyperbaric air became more in treating conditions other than decompression sickness, such as influenza, glaucoma, diabetes, pernicious anemia, arthritis, syphilis and certain cases of cancers. In retrospect, the sensitivity of malignant cells to high doses of Oxygen was tested. In 1943, De Almeida found that implanted tumors in rats were "softened" after repeated exposures to 6.0 ATA oxygen. For humans, Better results were achieved when combining radiation therapy and HBO at 3.0 ATA. Gradually, the effectiveness of the HBO in treating various medical conditions were investigated and proven till it reached to a confirmed list of indications nowadays. ${ }^{7}$

\section{Physiological basics of HBO therapy and methods of use}

There are two important factors that play a role in the physiological effectiveness of HBO. They are: the oxygen concentration and the applied pressure that should be greater than the ambient pressure. Oxygen is the key of living. It is normally $95 \%$ saturated hemoglobin at atmospheric pressure. Hundred milliliter of blood carries $19 \mathrm{ml}$ of oxygen in combination with hemoglobin, while $0.32 \mathrm{ml}$ is dissolved in plasma. When the inspired oxygen concentration increases to $100 \%$, subsequently the oxygen combination to hemoglobin increases to a maximum of $20 \mathrm{ml}$ and $2.09 \mathrm{ml}$ of dissolved oxygen in plasma. When the pressure is applied by 2 ATA to 3 ATA, the amount of oxygen carried in solution will increase to 4.4 to $6.8 \mathrm{ml}$ respectively, and that is sufficient to fulfill the tissue needs of oxygen without a contribution from oxygen bound to hemoglobin. This increased level of oxygen in plasma is responsible for most of the beneficial effects of HBO. ${ }^{1,8,9}$ HBO therapy can be delivered systemically or topically. Systemically, the patient is placed inside a chamber which is pressurized with compressed air and the patient inhales $100 \%$ oxygen through mask or hood. The average applied pressure is usually 2 to 4 ATA for 60$90 \mathrm{~min}$. The number of exposures may vary from 3 visits for acute simple conditions, up to 60 visits in severe, chronic conditions like the radiation illnesses. Topically, is that when topical oxygen dissolves in sufficient quantity, since the devices employed in the topical application of oxygen consist of a compartment to encase the affected portion of the body. The compartments that enclose the wound may be box-like or function as disposable plastic bags. ${ }^{1,9}$

\section{Approved applications of HBO therapy in medicine by UHMS}

In 2011, The UHMS has listed an updated 13 medical conditions, at which the research data and the clinical experience showed the effectiveness of HBO therapy in treating them. ${ }^{10}$ 


\section{Decompression illness: Air embolism and Decompression Sickness}

Air embolism, also called a gas embolism, occurs when an air bubble enters the vein or the artery and blocks it. It might be caused by sudden decompression, trauma, iatrogenic, intraoperative complications and miscellaneous and other rare causes. HBO at 3 ATA is considered the most effective treatment, because it reduces the size of air bubbles by about two-thirds and counteracts its secondary effects. The overall mortality of air embolism can be reduced from $30 \%$ to $14 \%$ if HBO therapy is given within 12 hours of the accident (Bercha et al 1996). Moreover, HBO has been used successfully in cases of air embolism in a complication of open heart surgery, endoscopy and transthoracic percutaneous thin-needle biopsy. ${ }^{11,12}$ It is also widely accepted that HBO is the only treatment for decompression sickness. For such cases, HBO should be started during the acute episode and continued till the symptoms are clear. ${ }^{13}$

\section{Carbon monoxide poisoning}

Although the recent technologies and the safety standards are more developed than before, 50,000 people in the United States each year visit the emergency department for carbon monoxide $\mathrm{CO}$ poisoning. Its common symptoms are headaches, nausea and vomiting, dizziness, general malaise, and altered mental status. Some patients may suffer from chest pain, shortness of breath and myocardial ischemia, and may require mechanical ventilation and treatment of shock. Individuals poisoned by $\mathrm{CO}$ often go on to develop neurological problems, including cognitive sequelae, anxiety and depression, persistent headaches, dizziness, sleep problems, motor weakness, vestibular and balance problems, gaze abnormalities, peripheral neuropathies, hearing loss, tinnitus and Parkinsonian-like syndrome. Oxygen is considered the standard treatment for $\mathrm{CO}$ poisoning to reverse its side effects. At high pressure (2.5 ATA), the oxygen is able to decrease the carboxyhaemoglobin half-life from 4-6 hrs to less than half hour. Moreover, $\mathrm{HBO}$ hastens $\mathrm{COHb}$ elimination, favorably modulates inflammatory processes, improves mitochondrial function, inhibits lipid peroxidation transiently, impairs leukocyte adhesion to injured microvasculature, and reduces brain inflammation caused by the COinduced adduct formation of myelin basic protein. Based upon three supportive randomized clinical trials in humans and considerable evidence from animal studies, HBO should be considered for all cases of acute symptomatic CO poisoning. ${ }^{1,14,15}$

\section{Clostridial myositis and myonecrosis (gas gangrene)}

Clostridial myositis and myonecrosis or gas gangrene is an acute, rapidly progressive, nonpyogenic, invasive clostridial infection of the muscles. They are characterized by profound toxemia, extensive edema, massive death of tissue and a variable degree of gas production. [16] HBO in combination with surgery and antibiotics is the preferred treatment. The acute problem of gas gangrene is the rapidly advancing phlegmon, which is caused by the continuous production of alpha toxin in infected but still viable tissue. The oxygen partial pressure of $250 \mathrm{mmHg}$ is necessary to stop toxin production completely, and the only way to achieve this is to start HBO therapy as soon as possible and then to continue therapy until the advance of the disease process has been clearly arrested. ${ }^{17}$

\section{Acute traumatic peripheral ischemia}

In severe trauma, as crush injury, blood circulation and thus healing may be compromised. This may lead to tissue necrosis and risk of extremity amputation. HBO has been successfully used as an adjunct therapy to surgery and antibiotics. Based on clinical and experimental evidence, $\mathrm{HBO}$ is able to minimize secondary complications and enhance healing in patients with acute traumatic peripheral ischemia cases. $^{18}$

\section{Enhancement of healing in selected problem wounds}

It was noticed that the wound heal faster when the patient moves from thin mountain air to a richer atmosphere and oxygen is considered as the most essential for healing process, hence the HBO has the ability to enhance the wound healing in diabetic and other nondiabetic wound problems. ${ }^{19} \mathrm{HBO}$ therapy enhances the oxygenation, decreases the edema and modifies the healing and immune responses. The use of HBO therapy is covered as adjunctive therapy only after there are no measurable signs of healing for at least 30 -days of treatment with standard wound therapy and must be used in addition to standard wound care..$^{20}$ Figure 1 , taken by Dr. Stoller, shows the effectiveness of $\mathrm{HBO}$ in treating a non-healing diabetic wound and proves the ability of $\mathrm{HBO}$ in saving up to $75 \%$ of diabetics from amputation. ${ }^{21}$
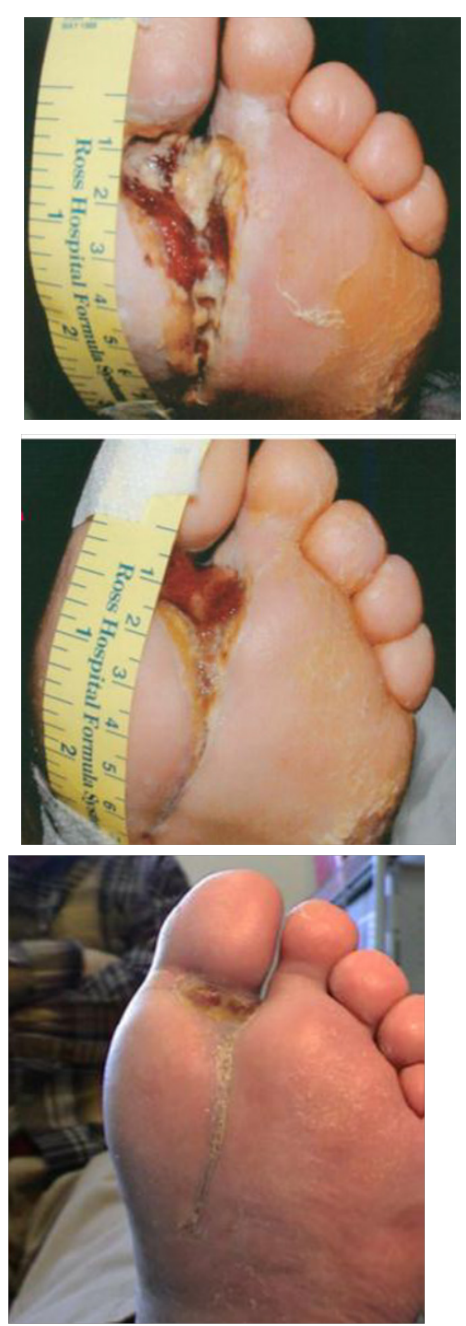

Figure $1 \mathrm{~A}$ : Wound present for one year and it was one day away from amputation; B: After 26 visits of $\mathrm{HBO}$ treatment, $100 \%$ oxygen at 2.4ATA; C: After 50 visits of $\mathrm{HBO}$ treatment, $100 \%$ oxygen at 2.4 ATA. $^{21}$ 


\section{Exceptional blood loss anemia}

HBO shows a good support for the basic metabolic needs of the tissue. This was found very helpful especially in patients who do not accept blood transfusion because of religious believes. ${ }^{22}$ The intermittent use HBO therapy at 3 ATA provides 6 volumes percent of oxygen in plasma that can be used directly by tissue cells. In addition, the number of stem cells released from bone marrow by 90 min oxygen therapy will increase by double. ${ }^{23}$

\section{Intracranial abscess}

HBO inhibits anaerobic bacteria, reduces cerebral edema, and modifies immune response. UHMS has recommended HBO for multiple, deep, or dominantly located abscesses. In addition, HBO can be administered for patients with severe infection who do not response to standard antibiotic treatment. The recommended treatment protocol for such cases is 1 or 2 sessions daily of HBO therapy at 2.0-2.5 ATA for 60-90 min each. ${ }^{1}$

\section{Necrotizing soft tissue infections}

In addition to surgical debridement and antibiotic coverage, $\mathrm{HBO}$ can be used as an adjunctive treatment for cases of necrotizing cellulitis, progressive dermal gangrene, anaerobic streptococcal myositis, crepitant anaerobic cellulitis, and necrotizing fasciitis. HBO increases tissue oxygen tension which enhances white cell killing of bacteria, inhibits growth of anaerobic organisms and increases the potentiality of oxidation reduction. ${ }^{1,9}$

\section{Refractory osteomyelitis}

Refractory osteomyelitis is defined as a chronic osteomyelitis that persists after appropriate interventions or where acute osteomyelitis has not responded to accepted management techniques. UHMS has recommended $\mathrm{HBO}$ as an adjunct treatment in addition to antibiotics, debridement, and nutritional support. The recommended protocol includes daily administration of HBO for 90-120 min at 2.0-2.5 ATA. Patients should be reviewed and re-evaluated after 40 sessions. ${ }^{1}$ In 2003, 11 of 14 patients with chronic refractory osteomyelitis were successfully treated with HBO therapy without any complications. ${ }^{24}$

\section{Skin flaps and grafts (compromised)}

Different clinical reports have shown that HBO can improve the survival/ the success of skin flaps and grafts. For such conditions, UHMS has recommended two sessions daily of HBO treatment at 2.0-2.5 ATA for 90-120 min each. Later on, when the graft or flap has become stabilized, the treatment can be reduced to one session daily and for another 20 days. $^{25}$

\section{Delayed radiation injury (soft tissue and bony necrosis)}

Radiation injuries include osteoradionecrosis, radiationcysitis and enteritis. Patients, who had exposed to 2000 to 5000 rads, may have difficulties in healing processes. This problem turns to be definite if the radiation dose is above 5000 rads. HBO is considered the keystone of treatment of radiation illness and it reaches to $94 \%$ success rate in the treatment of radionecrosis of the mandible injuries. ${ }^{26}$

\section{Thermal burns}

Several animal and human studies have supported the efficiency of using $\mathrm{HBO}$ as adjunctive treatment in thermal injuries. The reported benefits include: faster epithelialization, enhancement of PMN killing function, reduction of edema, preventing dermal ischemia, modulating the zone of stasis, preventing partial- to full-thickness conversion, preserving cellular metabolism and promoting of healing. The majority of clinical reports have shown reduction in mortality, time of hospital stay, number of surgeries and cost of care. Yet, careful patient selection is mandatory before choosing the HBO therapy. ${ }^{27,28}$

\section{Idiopathic sudden sensorineural hearing loss}

It has been approved by the board directors of UHMS on Oct 8, 2011, as an indication for HBO therapy. The most common clinical presentation involves an individual experiencing a sudden unilateral hearing loss, tinnitus, a sensation of aural fullness and vertigo. If the patient meets the selected criteria, then the recommended treatment profile consists of $100 \% \mathrm{O} 2$ at 2.0 to 2.5 atmospheres absolute for 90 minutes daily for 10 to 20 treatments. ${ }^{10}$

\section{Possible applications of HBO therapy in dentistry}

Hyperbaric oxygen therapy is considered a rapidly developing treatment modality in various fields of dentistry. Bone regeneration, osseointegration, treatment of periodontitis and treatment of osteoradionecrosis are some examples of possible applications. Yet, it needs further established evidences and clinical reports.

\section{Bone regeneration and its possible application in dentistry (implant, dry socket and fracture reunion)}

Multiple vitro studies have shown the good influence of HBO in bone regeneration through different physiological factors, as summarized in Figure 2.

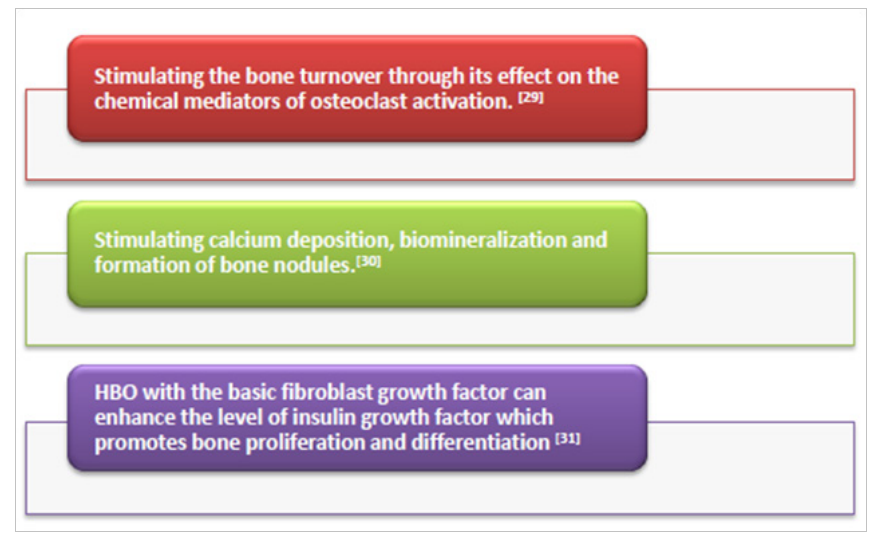

Figure 2 The effect of $\mathrm{HBO}$ on bone regeneration.

This suggests a promising potential application of HBO in:

1. Fracture healing and bone regeneration in the maxillofacial region.

2. Speeding the healing of alveolar bone after extraction, especially in smokers and diabetic patients to avoid alveolar osteitis (Figure 2). On the other hand, HBO has also shown an effect in the interface between the titanium implant and bone. A histomorphometric study indicated that HBO treatment was beneficial for the tissue incorporation of commercially pure titanium implants when placed immediately in free autogenous bone grafts ${ }^{29-32}$ It is also recommended for patients treated with more than 50 Grays, when implants were placed for more reliable healing. ${ }^{33}$ 


\section{Treatment of periodontitis}

Based on more than one study, shown in Table 1, the HBO has shown effectiveness as an adjunct therapy in the treatment of different types of periodontitis. Yet, follow up is suggested to ensure the length of the HBO effectiveness. 3-Treatment of Osteoradionecrosis and radiation illnesses in the maxiollofacial region: HBO is considered the keystone of treatment of radiation illness, since HBO therapy has reached up to $94 \%$ success rate in the treatment of radionecrosis of the mandible. ${ }^{26}$ It also has shown a significance as an adjunct therapy in the treatment of patients with various conditions of osteoreadionecrosis. ${ }^{37}$ Eleven out of 12 patients with refractory osteoradionecrosis of the mandible had excellent responses to HBO therapy which applied as an adjunct treatment by the department of oral and maxillofacial surgery at Wilford Hall USAF Medical center. The main conservative management for these patients included antibiotics, wound care and superficial sequestroctomy ${ }^{38}$ Other studies have shown that irradiation reduces the capacity of osseointegration around titanium implants. However, HBO therapy has been found to have positive effects on bone maturation after irradiation and may improve bone formation. ${ }^{39}$ Contraindications of HBO therapy There are 4 absolute contraindications and 10 relative contraindications that have been published by The Chinese Medical Association CMA published in 2013. The absolute contraindications are mainly pulmonary and hemorrhagic diseases. The relative contraindications should be used with caution due to possible side effects and they are mainly respiratory tract and sinus infection, high blood pressure, heart disease, the first trimester pregnancy, presence of tumor, retinal detachment and skull fracture (Table 2).$^{40}$ Oxygen toxicity Oxygen toxicity is a condition resulting from the harmful effects of breathing molecular oxygen at increased partial pressures. It is also known as oxygen toxicity syndrome. If HBOT is used for longer duration, sometimes even for short duration, it can lead to oxygen toxicity. The central nervous system and the pulmonary and ocular toxicity are the main and the most common complications. ${ }^{41}$ Ozone therapy vs. HBO therapy in wound healing.

Hyperbaric oxygen (HBO) therapy is based on ventilating the patient with $100 \%$ oxygen in pressure greater than the atmospheric pressure at sea level. HBO improves perfusion by increasing the level of free oxygen in the blood. Cell viability, energy production, and the production of collagen, which is important in wound healing. ${ }^{42}$ Ozone is a molecule consisting of three oxygen atoms. Ozone therapy is administration of a certain amount of oxygen/ozone mixture into body cavities or the circulatory system. Ozone reduces the amounts of antioxidants in plasma by activating them. The hydrogen peroxide that occurs is responsible for the biological and therapeutic effects of ozone. The decrease in the antioxidant level and stimulating effect of hydrogen peroxide generate a shock effect on the body in the ozone contacting process. As a result of this effect, a variety of defense systems, including primarily antioxidant enzyme expression, are stimulated. Thus, resistance to the oxidative processes increases. One of the first effects of hydrogen peroxide, which is thought to be one of the molecules responsible for the therapeutic activity of ozone, is slipping the hemoglobin-oxygen dissociation curve to the right and releasing oxygen to the tissues easily by increasing 2,3-diphosphoglycerate levels in red blood. ${ }^{43}$ Unfortunately, there is no credible, peer reviewed evidence to support the use of ozone as a type of medical therapy (Table 2). ${ }^{44}$

Table I The effect of HBO on the treatment of periodontitis

\begin{tabular}{llll}
\hline Study by & $\begin{array}{l}\text { Type of the } \\
\text { study }\end{array}$ & $\begin{array}{l}\text { Periodontal } \\
\text { condition }\end{array}$ & Main findings \\
\hline${\text { Chen TL et al. }{ }^{34}}^{\text {Case- Control }}$ & $\begin{array}{l}\text { Aggressive } \\
\text { study }\end{array}$ & $\begin{array}{l}\text { HBO therapy combined with scaling and root planning (SRP) was the most } \\
\text { beneficial in the treatment of AP.The effect could last more than two years. }\end{array}$ \\
$\begin{array}{l}\text { Nogueira GR } \\
\text { et al. }{ }^{35}\end{array}$ & $\begin{array}{l}\text { Case- Control } \\
\text { study }\end{array}$ & $\begin{array}{l}\text { Chronic } \\
\text { periodontitis }\end{array}$ & $\begin{array}{l}\text { HBO therapy with SPR showed greater probing reduction and attachment gain } \\
\text { than SRP alone. Yet, it had a short-term beneficial effect and may be considered a } \\
\text { potential adjunctive therapy. }{ }^{35}\end{array}$ \\
$\begin{array}{l}\text { Signoretto CA } \\
\text { et al. }{ }^{36}\end{array}$ & Vitro study & $\begin{array}{l}\text { Chronic } \\
\text { periodontitis }\end{array}$ & $\begin{array}{l}\text { HBO with SRP reduced (by up to } 99.9 \%) \text { the gram-negative anaerobe loads of the } \\
\text { subgingival microflora for } 3 \text { months at least. }\end{array}$ \\
\hline
\end{tabular}

Table 2 Contraindications of HBO

\begin{tabular}{l} 
Absolute contraindications \\
\hline Untreated pneumothorax and pneumomediastinum \\
Pulmonary bulla \\
Active hemorrhage and hemorrhagic disease \\
The formation of tuberculous cavity and hemoptysis \\
The tension pneumothorax without treatment
\end{tabular}

\author{
Relative contraindications \\ Severe upper respiratory tract infection \\ Severe emphysema \\ Bronchiectasis disease \\ Sinus infection \\ All degrees of atrioventricular blo \\ High blood pressure $(<160 / 100 \mathrm{~mm} \mathrm{Hg})$ \\ Untreated malignant tumor \\ Retinal detachment \\ The early stage of pregnancy (3 months) \\ Bradycardia (> 50 times/min) \\ Intraventricular external drainage \\ Fracture of the skull base with cerebrospinal fluid leakage \\ Birth weight $>2000 \mathrm{~g}$ in premature and low birth weight infants \\ Serious infection of the upper respiratory tract \\ High blood pressure (SBP< $180 \mathrm{~mm} \mathrm{Hg}, \mathrm{DBP}<110 \mathrm{~mm} \mathrm{Hg})$ \\ Patients with chronic obstructive pulmonary disease with $\mathrm{CO}_{2}$ retention
}




\section{Discussion and conclusion}

Last but not least, the applications of HBO therapy are getting wider in the medical and the dental field for achieving better prognosis. It could have a promising future in the dental field to be served topically for treating many challenging cases, such as; the non-healing ulcers, peri-implantitis, dry sockets, osteochemonecrosis, and increase the success rate of healing in heavy smokers and uncontrolled diabetics. Yet, despite of the great effectiveness of HBO that have been detected, there are some reported complications which require a careful preexamination and monitoring. The predominant complication is represented by pressure equalization problems within the middle ear, while serious complications rarely occur. ${ }^{45}$ On the other hand, nonemergent patients who are treated routinely with hyperbaric oxygen, via a head hood, have a potential risk of CNS oxygen toxicity which is three fold greater than historical reports and in agreement with more recent reports. The reason for this apparent increase in incidence of CNS oxygen toxicity is unknown. But the complications which were observed were transient and they were limited mostly within the duration of the treatment. ${ }^{46,47}$ In conclusion, the studies of HBO are increasing year by year worldwide, due to many previous studies that have shown positive results and encouraged the others to investigate more in the benefits of HBO. As dentists, we hope to reach to the level of approved dental indications of HBO by UHMS based on strong scientific evidences.

\section{Acknowledgment}

None.

\section{Conflict of interest}

None.

\section{References}

1. Hampson NB, Hyperbaric Oxygen Therapy: 1999 Committee report. Kensington MD, Undersea and Hyperbaric Medical Society, 1999.

2. Hunt TK. The physiology of wound healing. Ann Emerg Med. 1988;17(12):1265-73.

3. Kinghton DR, Silver IA, Hunt TK. Regulation of wound healing angiogenesis-effect of oxygen gradients and inspired oxygen concentration. Surgery. 1981;90(2):262-70.

4. Kinghton DR, Halliday B, Hunt TK. Oxygen as an antibiotic: the effect of inspired oxygen on infection. Arch Surg. 1984;119(2):199-204.

5. Mader JT, Adams KR, Couch LA, et al. Potentiation of tobramycin by hyperbaric oxygen in experimental Pseudomonas aeruginosa osteomyelitis (Abstract 1331). Abstracts of the 27 $7^{\text {th }}$ Interscience Conference on Antimicrobial Agents and Chemotherapy. Washington, DC, American Society for Microbiology, 1987.

6. Henshaw N. Aero-Chalinos. Dublin, Dancer, 1664.

7. Neuman T, Thomas S. Physiology and Medicine of Hyperbaric Oxygen Therapy. Philadelphia. Sannders Elsevier;2008

8. Bassett BE, Bennett PB. Introduction to the physical and physiological basis of hyperbaric therapy. Undersea and Hyperbaric Medical Society. $1986 ; 11-24$

9. Tibbles PM, Edelsberg JS. Hyperbaric oxygen therapy. NEJM. 1996:1642-8.

10. $H B O$ indications. (2017).
11. Jain.K. Textbook of Hyperbaric Medicine. Germany: Hogrefe \& Huber;2004

12. Branger AB, Lambersten CJ, Eckmann DM. Cerbral gas embolism absorption. Anesth Analg. 1997;84;1203-7.

13. Moon RE, Sheffield PJ. Guidelines for treatment of decompression illness. Aviat Apace EnvironMed. 1997;68:234-43.

14. Weaver LK. Hyperbaric Oxygen therapy for carbon monoxide poisoning. Undersea Hyperb Med. 2014;41(4):339-54.

15. Pace N, Strajman E, Walker E. Acceleration of carbon monoxide elimination in man by high pressure oxygen. Science. 1950;111(2894):652-4.

16. Stevens DL. The pathogenesis of clostridial myonecrosis. Int J Microbial. 2000;290(4-5):497-502.

17. Van Unnik AJM. Inhibition of toxin production in Clostridium perfringens in vitro by hyperbaric oxygen.Antonie Leeuwonhoek Microbial. 1965;31(1):181-186.

18. Greensmith J. Hyperbaric Oxygen Therapy in Extremity Trauma: Prospective on modern orthopaedics. $J$ Am Acad Orthop Surg. 2004;12(6):376-384.

19. Ladizinsky D, R David. New insights into oxygen therapy for wound healing. Wounds. 2010;22(12):294-300

20. UHC MA Coverage Summary: Hyperbaric Oxygen Therapy Confidential and Proprietary, (OUnitedHealth care, Inc. 2016.

21. Hyperbaric Medical Center of New Mexico.

22. Hart G. Exceptional blood loss anemia treatment with hyperbaric oxygen. JAMA. 1974;228(8):1028-1029.

23. Blood Loss and Anemia, Canada.

24. Chen CE, Shih ST, Fu TH, et al. Hyperbaric Oxygen Therapy in the Treatment of Chronic Refractory Osteomyelitis: A Preliminary Report. Chang Gung Med J. 2003:26(2):114-21.

25. Perrins DJD. Influence of hyperbaric oxygen on the survival of split skin grafts. Lancet. 1967;1(7495):868-71.

26. Sahni T, Singh P, John MJ. Hyperbaric oxygen therapy: current trends and applications. J Assoc Physicians India. 2003;51:280-4.

27. Cianci P, Stato R. Adjunctive hyperbaric oxygen therapy in the treatment of thermal burns: a review. Burns. 1994;20:5-14.

28. Cianci P, Slade JB, Sato RM, et al. Adjunctive hyperbaric oxygen therapy in the treatment of thermal burns. Undersea Hyperb Med. 2013;40(1):89-108.

29. Sugi T, Sasaki M, Yamashiro T, et al. Hyperbaric Oxygen stimulates the proliferation and differentiation of Raw246.7 cells, Open Journal of Stomatology. 2015;5(12):309-320.

30. Wu, Dong, Malda, Jos, Crawford, et al. Effects of hyperbaric oxygen on proliferation and differenciation of osteoblasts derived from human alveolar bone. Connec Tissue Res. 2007;48(4):206-13.

31. Johnsson K, Hansson A, Granström G, et al. The effects of hyperbaric oxygenation on bone-titanium implant interface strength with and without preceding irradiation. Int J Oral Maxillofac Implants. 1993;8(4):415-9.

32. Sawai T, Niimi A, Johansson CB, et al. The effect of hyperbaric oxygen treatment on bone tissue reactions to c.p. titanium implants placed in free autogenous bone grafts. A histomorphometric study in the rabbit mandible. Clin Oral Implants Res. 1998;9(6):384-97.

33. Taylor TD, Worthington P. Osseointegrated implant rehabilitation of the 
previously irradiated mandible: Results of a limited trial at 3 to 7 years. $J$ Prosthet Dent 1993;69(1):60-9.

34. Chen TL, Xu B, Liu JC, et al. Effects of hyperbaric oxygen on aggressive periodontitis and subgingival anaerobes in Chinese patients. $J$ Indian Soc Periodontol. 2012;16(4):492-7.

35. Nogueira-Filho GR, Rosa BT, David-Neto JR. Effects of hyperbaric oxygen therapy on the treatment of severe cases of periodontitis. Undersea Hyperb Med. 2010;37(2):107-14.

36. Signoretto C, Bianchi F, Burlacchini G, et al. Microbiological evaluation of the effects of hyperbaric oxygen on periodontal disease. New Microbiol. 2007;30(4):431-7.

37. Sharma W. Role of hyperbaric oxygen therapy in dental surgery. IJASM 2003:47(1):23-29.

38. Mansfiels M, Sanders D, Heinbach R, et al. Hyperbaric Oxygen as an adjunct in the treatment of osteoradionecrosis of the mandible. J Oral Surgery. 1981;39.

39. Johnsson Ase A, Sawaii Toshihiro, Jacobsun Magnus, et al. A histomorphometric study of bone reactions to titanium implants in irradiated bone and the effect of hyperbaric oxygen treatment. The International Journal of Oral and Maxillofacial Implants. 1999;14:699_ 706.
40. Yan L, Liang T, Cheng O. Hyperbaric oxygen therapy in China. Med Gas Res. 2015;5:3

41. Patel D, Goel A, Agarwal SB, et al. Oxygen Toxicity. JIACM. 2003;4(3):234-7.

42. Feldmeier JJ. Hyperbaric oxygen 2003: Indications and results: The Hyperbaric Oxygen Therapy Committee Report. Undersea and Hyperbaric Medical Society Inc. Kensingto;2003.

43. Signoretto C, Bianchi F, Burlacchini G, et al. Microbiological evaluation of the effects of hyperbaric oxygen on periodontal disease. New Microbiol. 2007;30(4):431-7.

44. Oxygen Therapy. American Cancer Society;2012.

45. Plafki C, Peters $\mathrm{P}$, Almeling M, et al. Complications and side effects of hyperbaric oxygen therapy. Aviat Space Environ Med.2000;71(2):119-24.

46. Hampson N, Atik D. Central nervous system oxygen toxicity during routine hyperbaric oxygen therapy. Undersea Hyperb Med. 2003;30(2):147-53.

47. Devaraj D, Srisakthi D. Hyperbaric Oxygen Therapy - Can It Be the New Era in Dentistry? J Clin Diagn Res. 2014;8(2):263-265. 